This item was submitted to Loughborough's Research Repository by the author.

Items in Figshare are protected by copyright, with all rights reserved, unless otherwise indicated.

\title{
Acute aerobic exercise induces a preferential mobilisation of plasmacytoid dendritic cells into the peripheral blood in man
}

\section{PLEASE CITE THE PUBLISHED VERSION}

https://doi.org/10.1016/j.physbeh.2018.05.012

\section{PUBLISHER}

(C) Elsevier

VERSION

AM (Accepted Manuscript)

\section{PUBLISHER STATEMENT}

This work is made available according to the conditions of the Creative Commons Attribution-NonCommercialNoDerivatives 4.0 International (CC BY-NC-ND 4.0) licence. Full details of this licence are available at: https://creativecommons.org/licenses/by-nc-nd/4.0/

\section{LICENCE}

CC BY-NC-ND 4.0

\section{REPOSITORY RECORD}

Brown, Frankie F., John P. Campbell, Alexander J. Wadley, James P. Fisher, Sarah Aldred, and James E. Turner. 2019. "Acute Aerobic Exercise Induces a Preferential Mobilisation of Plasmacytoid Dendritic Cells into the Peripheral Blood in Man". figshare. https://hdl.handle.net/2134/33277. 


\section{Acute aerobic exercise induces a preferential mobilisation of plasmacytoid dendritic cells}

2 into the peripheral blood in man.

$4 \quad{ }^{1}$ FRANKIE F. BROWN ${ }^{\mathrm{a}},{ }^{1}$ JOHN P. CAMPBELL ${ }^{\mathrm{a}, \mathrm{b}}$, ALEX J. WADLEY $^{\mathrm{c}}$, JAMES P. FISHER ${ }^{\mathrm{d}}$, 5 SARAH ALDRED ${ }^{\mathrm{d}}, \&$ JAMES E. TURNER ${ }^{\mathrm{a}} *$

6

$7 \quad{ }^{a}$ Department for Health, University of Bath, Bath, UK

$8 \quad{ }^{\mathrm{b}}$ Clinical Immunology, University of Birmingham, Birmingham, UK

$9{ }^{\mathrm{c}}$ School Sport, Exercise \& Health Sciences, Loughborough University, Loughborough, LE11 3TU,

$10 U K$

$11{ }^{d}$ School of Sport, Exercise and Rehabilitation Sciences, University of Birmingham, Birmingham, $12 U K$

13

14

15

$16{ }^{1}$ These authors contributed equally to this work and should be considered as co-first authors

$18 *$ Corresponding author:

19 Address: Dr James E Turner. Department for Health, University of Bath, Claverton Down, Bath, 20 BA2 7AY. Tel: 0122538 3566. E-mail: j.e.turner@bath.ac.uk

21

22 Key words: dendritic cells, myeloid, plasmacytoid, exercise, humans 23 


\section{Abstract}

25 Dendritic cells (DCs) are important sentinel cells of the immune system responsible for

26 presenting antigen to $\mathrm{T}$ cells. Exercise is known to cause an acute and transient increase in the

27 frequency of DCs in the bloodstream in humans, yet there are contradictory findings in the

28 literature regarding the phenotypic composition of DCs mobilised during exercise, which may

29 have implications for immune regulation and health. Accordingly, we sought to investigate the

30 composition of DC sub-populations mobilised in response to acute aerobic exercise. Nine

31 healthy males (age, $21.9 \pm 3.6$ years; height, $177.8 \pm 5.4 \mathrm{~cm}$; body mass, $78.9 \pm 10.8 \mathrm{~kg}$; body

32 mass index, $24.9 \pm 3.3 \mathrm{~kg} \cdot \mathrm{m}^{2}$; $\dot{\mathrm{VO}}_{2 \mathrm{MAx}}, 41.5 \pm 5.1 \mathrm{~mL} \cdot \mathrm{kg} \cdot \mathrm{min}^{-1}$ ) cycled for 20 minutes at $80 \%$

$33 \dot{\mathrm{VO}}_{2 \text { MAX. }}$. Blood was sampled at baseline, during the final minute of exercise and 30 minutes

34 later. Using flow cytometry, total DCs were defined as Lineage- (CD3, CD19, CD20, CD14,

35 CD56) HLA-DR+ and subsequently identified as plasmacytoid DCs (CD303+) and myeloid DCs

36 (CD303-). Myeloid DCs were analysed for expression of CD1c and CD141 to yield four sub-

37 populations; $\mathrm{CD} 1 \mathrm{c}-\mathrm{CD} 141+$; $\mathrm{CD} 1 \mathrm{c}+\mathrm{CD} 141+$; CD1c+CD141- and CD1c-CD141-. Expression

38 of CD205 was also analysed on all DC sub-populations to identify DCs capable of recognising

39 apoptotic and necrotic cells. Total DCs increased by $150 \%$ during exercise $\left(F_{(1,10)}=60 ; p<0.05\right.$,

$\left.40 \eta^{2}=0.9\right)$. Plasmacytoid DCs mobilised to a greater magnitude than myeloid DCs $(195 \pm 131 \%$ vs.

$41131 \pm 100 \%$; $\mathrm{p}<.05)$. Among myeloid DCs, CD1c-CD141- cells showed the largest exercise-

42 induced mobilisation $(167 \pm 122 \%)$, with a stepwise pattern observed among the remaining sub-

43 populations: $\mathrm{CD} 1 \mathrm{c}+\mathrm{CD} 141-(79 \pm 50 \%)$, followed by $\mathrm{CD} 1 \mathrm{c}+\mathrm{CD} 141+(44 \pm 41 \%)$, with the

44 smallest response shown by CD1c-CD141+ cells $(23 \pm 54 \%)(\mathrm{p}<.05)$. Among myeloid DCs,

45 CD205- cells were the most exercise responsive. All DC subsets returned to resting levels within

4630 minutes of exercise cessation. These results show that there is a preferential mobilisation of 
47 plasmacytoid DCs during exercise. Given the functional repertoire of plasmacytoid DCs, which

48 includes the production of interferons against viral and bacterial pathogens, these findings

49 indicate that exercise may augment immune-surveillance by preferentially mobilising effector

50 cells; these findings have general implications for the promotion of exercise for health, and

51 specifically for the optimisation of DC harvest for cancer immunotherapy.

52 


\section{1. Introduction}

54 Acute aerobic exercise causes profound alterations to the cellular composition of

55 peripheral blood, whereby the frequency of many leukocyte subsets increases during exercise,

56 followed by a decline in the hours after [1]. For many types of immune cell subsets, the

57 magnitude of change in response to exercise is usually largest among cells with the strongest

58 effector potential [2-4]. Accordingly, this exercise-induced effect is considered a conserved

59 evolutionary response which causes the redistribution of effector cells to peripheral tissues to

60 conduct immune-surveillance [5]. Cells of a lymphoid lineage, such as T cells [2, 6] and natural

61 killer (NK) cells [7], are the most widely researched. Cells with myeloid characteristics have

62 received less attention in the exercise literature, except for a limited number of studies which

63 have examined monocytes [8-10]. For example, it has been shown that alternatively-activated

64 M2-like monocytes preferentially mobilise into blood during exercise $[8,9]$, whereas other work

65 has shown that the most exercise responsive cells are classically-activated M1-like monocytes

66 [10]. Studies examining the mobilisation patterns of dendritic cell (DC) subsets in response to

67 exercise have provided equivocal evidence, despite the critical role DCs play in initiating and

68 directing immune responses.

69 DCs are often considered tissue resident cells, but these sentinels of the immune system,

70 consist of multiple sub-populations with unique functions, and many DC subsets are found

71 transmigrating between peripheral blood and the lymphatic system [11]. The central function of

72 these professional antigen-presenting cells (APCs) is to ingest pathogens or debris from

73 apoptotic or necrotic cells, and subsequently process and present antigen to lymphocytes [11].

74 DCs also help to regulate the immune response through co-stimulatory or co-inhibitory

75 molecules $[11,12]$. The two major sub-populations of DCs are myeloid DCs and plasmacytoid 
76 DCs [13]. Some studies have shown that immediately after 15-20 minutes of moderate intensity

77 exercise, total DC numbers increase in blood $[14,15]$ with a preferential increase in

78 plasmacytoid DCs [16]. However, other studies have shown that after more prolonged exercise,

79 such as a marathon, myeloid DCs increase but plasmacytoid DCs may decrease immediately

80 post-exercise $[17,18]$. In light of these contradictory findings, further investigation of the DC

81 response to exercise is warranted. In addition, greater clarity on the phenotypic composition of

82 plasmacytoid and myeloid DCs mobilised during exercise in healthy adults is needed to provide

83 insight into the functional and homing characteristics of exercise-responsive DCs.

84 DCs express high levels of MHC class II (HLA-DR) and do not express other lineage

85 markers expressed on monocytes, T cells, B cells and NK cells, and are therefore referred to as

86 being Lineage- $(\mathrm{CD} 3, \mathrm{CD} 19, \mathrm{CD} 20, \mathrm{CD} 14, \mathrm{CD} 56) \mathrm{HLA}-\mathrm{DR}+[13,19]$. Expression of the cell

87 surface protein CD303 enables further differentiation of plasmacytoid (CD303+) and myeloid

88 DCs (CD303-) [20]. Among myeloid cells, four sub-populations can be identified based on

89 CD1c and CD141 expression [21-24] (Table 1). Other cell-surface proteins, such as co-

90 stimulatory or co-inhibitory molecules, can indicate the functional characteristics of DCs, for

91 example receptors such as CD205 (also known as DEC-205) [25] which enables recognition of

92 apoptotic or necrotic cells [26]. Another commonly assessed cell-surface receptor expressed on

93 activated DCs is CD209 (also known as DC-SIGN) which recognises a wide array of ligands

94 from viruses and bacteria, and is also involved in adhesion, migration, signalling and antigen

95 presentation [27]. To date, the effect of exercise on DCs that express these functional markers is

96 not known.

97 Clarifying the exercise-induced kinetics of DCs is important because it has been proposed

98 that acute bouts of vigorous steady state exercise may be a strategy to optimise immune 
99 competency, for example, by enhancing vaccination responses [28-31]. Additionally, it has

100 recently been proposed that exercise could be a powerful means of increasing peripheral blood

101 mononuclear cell yields for the purposes of immunotherapy [32, 33]. To date, the most targeted

102 malignancies for DC immunotherapies are melanoma, prostate cancer, glioblastoma and renal

103 cell carcinoma, but trials are being conducted with many other cancers [34, 35]. A common

104 approach is to isolate peripheral blood mononuclear cells from patients to generate monocyte-

105 derived DCs ex vivo with growth factors and antigen stimulation, before re-administering the cell

106 preparations $[34,36]$. There are several examples of clinically effective DC immunotherapy

107 regimens, but methodologies continue to be adapted and improved, with recent emphasis on

108 harvesting DC sub-populations directly from blood, with a particular focus on either

109 plasmacytoid DCs due to their effector potential, or CD1c+ and/or CD141+ myeloid subsets for

110 their ability to cross present antigen to cytotoxic $\mathrm{CD} 8+\mathrm{T}$ cells $[34,36]$. Thus, if adjunctive

111 strategies such as exercise are employed to improve cell yields for DC immunotherapy, it is

112 important to understand how naturally occurring DC sub-populations respond to exercise-

113 induced stimulation. Therefore, the aim of this study was to conduct a detailed immuno-

114 phenotypic analyses of DC sub-populations present in peripheral blood before, during and after

115 an acute bout of vigorous steady state aerobic exercise. 


\section{2. Methods}

\section{2.1. Participants}

118 Nine healthy men were included in the present analyses (age, $21.9 \pm 3.6$ years; height, $177.8 \pm$

$1195.4 \mathrm{~cm}$; body mass, $78.9 \pm 10.8 \mathrm{~kg}$; body mass index, $24.9 \pm 3.3 \mathrm{~kg} . \mathrm{m}^{2} ; \dot{\mathrm{VO}}_{2 \mathrm{MAx}}, 41.5 \pm 5.1$

$120 \mathrm{~mL} \cdot \mathrm{kg} \cdot \mathrm{min}^{-1}$ ) (ethical approval reference: ERN_12-0830; University of Birmingham, UK).

121 These nine participants represent a sub-group from a total of ten men who took part in other

122 investigations [37-40] with peripheral blood mononuclear cells (PBMCs) that were available for

123 analysis following cryopreservation.

\section{2.2. Pre-experimental procedures}

126 Height and body mass were assessed using standard methods and cardiorespiratory fitness $\left(\dot{\mathrm{V}}_{2}\right.$

127 MAX) was measured on a cycle ergometer. Expired air samples were assessed for oxygen

128 consumption and carbon dioxide production using breath-by-breath analysis, with heart rate

129 monitored via telemetry, and ratings of perceived exertion recorded using the Borg scale [37-40].

130

\section{2.3. Exercise trial and blood sampling}

132 At least seven days after preliminary measurements, and following an overnight fast, participants

133 reported to the laboratory in the morning, and a blood sample was collected from a cannulated

134 forearm vein after a 15-minute seated rest (baseline). The exercise trial consisted of steady state

135 cycling at $80 \% \dot{\mathrm{VO}}_{2}$ MAX for 20 minutes, at a power output determined from the $\dot{\mathrm{VO}}_{2}$ MAX test.

136 Exercise intensity was monitored with breath-by-breath measurements. Heart rate and ratings of 137 perceived exertion were recorded throughout the exercise trial. A second blood sample was 
138 collected in the final minute of exercise at $80 \% \dot{\mathrm{V}}_{2 \mathrm{MAX}}$ (exercise) and a third blood sample

139 collected post-exercise, after 30 minutes of seated rest (+30 minutes) [37-40].

140

141 2.4. Peripheral blood mononuclear cell (PBMC) isolation

142 Blood with potassium ethylene-diamine-tetra-acetic acid (EDTA) as an anticoagulant was diluted

143 1:1 with Roswell Park Memorial Institute Media (RPMI), and layered on top of Ficoll paque

144 PLUS (GE Healthcare) (2 blood:1 Ficoll), before centrifuging at $400 \times \mathrm{g}$ for 30 minutes at $21^{\circ} \mathrm{C}$.

145 PBMCs were aspirated and washed three times in RPMI by centrifuging at $200 \times \mathrm{g}$ for 5

146 minutes. Cells were re-suspended in freezing mixture (70\% RPMI, 20\% fetal calf serum (FCS)

147 and $10 \%$ dimethyl sulfoxide (DMSO)) and frozen at $-1{ }^{\circ} \mathrm{C} / \mathrm{min}$ using a freezing container

148 (Nalgene 'Mr Frosty' Thermoscientific). Cells were stored at $-80^{\circ} \mathrm{C}$ and analysed within six

149 months $[39,40]$.

150

151 2.5. Flow cytometry

152 PBMCs were thawed rapidly at $37^{\circ} \mathrm{C}$ and washed twice in phosphate buffered saline (PBS)

153 containing $2 \% \mathrm{FCS}$ and $2 \mathrm{mM}$ EDTA by centrifuging at $400 \times \mathrm{g}$ for 5 minutes. PBMCs were

154 counted using a haemocytometer and approximately 300,000 cells were added to tubes for

155 incubation with fluorescently conjugated antibodies to identify DCs and sub-populations using

156 eight-colour flow cytometry (FACS-CANTO, Becton-Dickenson, San Jose, USA). The

157 following monoclonal antibodies were used: FITC-conjugated anti-Lineage 2 cocktail (CD3

158 clone \# SK7, CD19 clone \# SJ25C1, CD20 clone \# L27, CD14 clone \# M 9 P9, CD56 clone \#

159 NCAM 16.2), V500-conjugated anti-HLA-DR clone \# G46-6, V450-conjugated anti-CD209

160 clone \# DCN46 (BD Biosciences, San Diego, USA), APC-conjugated anti-CD303 clone \# 201A, 
161 PE-Cy7-conjugated anti-CD141 clone \# M80, APC-Cy7-conjugated anti-CD1c clone \# L161

162 (BioLegend, San Diego, USA), PE-conjugated anti-CD205 clone \# MG38 (BD Pharmingen, San

163 Diego, USA). In addition, 7-aminoactinomycin D (7-AAD) (BD Pharmingen, San Diego, USA)

164 was used to exclude necrotic and apoptotic cells. Fluorescence-minus-one (FMO) tubes

165 established negative and positive gating strategies for CD205 and CD209 expression (data not 166 shown).

168 2.6. Flow cytometry analysis

169 Data were analysed using FlowJo version Xv 0.7 (Tree Star, Inc., Ashland, OR). Doublets were

170 excluded by gating forward versus forward-scatter. PBMCs were gated on the forward versus

171 side-scatter. Dead cells were excluded by gating 7AAD versus side-scatter. Total DCs were

172 identified as being Lineage-HLA-DR+, and analysed for expression of CD303 to identify

173 plasmacytoid DCs (Lineage-HLA-DR+CD303+) and myeloid DCs (Lineage-HLA-

174 DR+CD303-). Myeloid DCs were analysed for expression of CD1c and CD141 to yield four

175 sub-populations: CD1c-CD141-; CD1c+CD141-; CD1c+CD141+; CD1c-CD141+ (Table 1

176 and Figure 1). All cell populations were examined for expression of CD205 and CD209. The

177 absolute number of DCs and sub-populations was determined from the PBMC count (Coulter

$178 \mathrm{ACT}^{\text {diff }}$ haematology analyser, Beckman-Coulter, High Wycombe, UK).

179

180 2.7. Statistical analyses

181 Data were inspected for normal distribution using the Shapiro-Wilk test. Non-normally

182 distributed data were transformed logarithmically. Responses to exercise were examined using

183 repeated-measures Analyses of Variance (ANOVA). When data violated sphericity, a 
184 Greenhouse-Geisser correction was applied. Differences between individual time points were 185 examined using post-hoc paired samples $t$-tests. Statistical significance was accepted at the $p<.05$ 186 level. Data are presented as means \pm standard deviation (SD) unless otherwise stated. Data were 187 analysed using SPSS statistical package version 22 (SPSS Inc, USA). 
189 3.1. Exercise trial

190 All participants completed the exercise trial [38]. The mean intensity of exercise was $80 \pm 6 \%$ of

$191 \dot{\mathrm{VO}}_{2 \mathrm{MAX}}$, average ratings of perceived exertion were $16 \pm 1$, and the mean heart rate throughout

192 the exercise task was $176 \pm 7$ beats per minute representing $91 \pm 3 \%$ of measured maximum

193 heart rate.

194

195 3.2. DCs

196 Total leukocytes, lymphocytes and monocytes exhibited the characteristic exercise-induced

197 changes as reported previously [39, 40]. DCs (Lineage-HLA-DR+) increased in numbers in

198 peripheral blood with exercise by approximately $150 \%$, returning to baseline levels within 30

199 minutes (main effect of time; $\left.F_{(1,10)}=60 ; p<0.05 \eta^{2}=0.9\right)$ (Figure 2a and 2b).

200

201 3.3. DC sub-populations

202 All DC sub-populations exhibited a statistically significant increase in cell numbers during

203 exercise, except for CD1c-CD141+ myeloid DCs, and returned to pre-exercise levels within 30

204 minutes of exercise cessation (Table 2). Overall, plasmacytoid DCs mobilised to a greater

205 magnitude than myeloid DCs ( $195 \pm 131 \%$ vs. $131 \pm 100 \%$; $p<.05$; Figure $2 b)$. Among

206 myeloid DCs, CD1c-CD141- showed the largest magnitude of exercise-induced change (167 \pm

$207122 \%$ ) with a stepwise mobilisation pattern among remaining sub-populations: CD1c+ CD141-

$208(79 \pm 50 \%)$, followed by CD1c+CD141+ $(44 \pm 41 \%)$ with the smallest response shown by

209 CD1c-CD141+ cells $(23 \pm 54 \%) p<.05$. 
211 3.4. DCs and sub-populations expressing CD205 and analysis of CD205 cell-surface expression

212 density

213 The majority of DCs and sub-populations were CD205+ (mean \pm SD; $97.8 \pm 3.6 \%$; across all

214 sub-populations and participants) [13]. There were no differences in the proportion of DCs

215 expressing CD205+ between the different sub-populations (data not shown). Generally, CD205+

216 and CD205- cells among all sub-populations mobilised into blood during exercise, returning to

217 baseline levels within 30 minutes of exercise (Table 2 and Figure 3). However, there was a trend

218 for a larger mobilisation of CD205- cells in the majority of sub-populations (Figure 3).

219 Compared to CD205+ cells, a larger and statistically significant mobilisation of CD205- cells

220 was observed among CD1c-CD141+ cells and the CD1c+CD141+ cells $(p$ 's $<.05$; Figure $3 \mathrm{e}$

221 and 3f). For example, CD1c-CD141+CD205- cells and CD1c+CD141+CD205- cells exhibited

222 a mobilisation that was $80 \%$ and $70 \%$ greater than their CD205+ counterparts. Different to other

223 cells, plasmacytoid DCs exhibited a trend for a larger mobilisation of CD205+ cells (Figure 3c).

224 We also examined whether exercise altered the cell-surface expression density of CD205. At

225 baseline, the cell-surface expression density of CD205 was greater in the three myeloid sub-

226 populations; $\mathrm{CD} 1 \mathrm{c}-\mathrm{CD} 141+$ and $\mathrm{CD} 1 \mathrm{c}+\mathrm{CD} 141+$ and $\mathrm{CD} 1 \mathrm{c}+\mathrm{CD} 141-$ compared to

227 CD1c-CD141- and plasmacytoid DCs (data not shown). In addition, CD205 expression density

228 did not change in response to exercise (data not shown).

229

230 3.5. DCs and sub-populations expressing CD209 and analysis of CD209 cell-surface expression 231 density 
232 DCs and their sub-populations did not express CD209 and there were no changes in the numbers 233 or proportions of CD209- cells, or alterations in the cell-surface expression density of CD209 in 234 response to exercise (data not shown). 


\section{Discussion}

The present study demonstrates that the total number of DCs increased in peripheral

237 blood during exercise by $150 \%$ and among the major DC sub-populations, plasmacytoid DCs

238 mobilised by $195 \%$ whereas myeloid DCs exhibited a smaller increase of $131 \%$. We show for

239 the first time, that among the four sub-populations of myeloid DCs, there was a stepwise

240 mobilisation pattern: a $167 \%$ increase with CD1c-CD141- cells, a 79\% increase with

$241 \mathrm{CD} 1 \mathrm{c}+\mathrm{CD} 141-$ cells, a $44 \%$ increase with CD1c $+\mathrm{CD} 141+$ cells and a $23 \%$ increase with

242 CD1c-CD141+ cells.

243 To date, the phenotypic characteristics of DC kinetics during exercise remains unclear as

244 only a limited number of studies have investigated the mobilisation of DC sub-populations in

245 response to physical stressors, and these studies have produced seemingly contradictory findings.

246 In the study herein, we show that both major DC subsets increase during exercise, with a greater

247 mobilisation response observed among plasmacytoid DCs compared to myeloid DCs. In

248 agreement with these results, a large and preferential exercise-induced mobilisation of

249 plasmacytoid DCs (200\% increase) compared to myeloid DCs (100\% increase) has also been

250 reported by a study that collected blood samples after vigorous ice hockey [16]. Contradicting

251 these findings, in two studies it has been shown that plasmacytoid DCs may decrease

252 immediately after long-duration exercise $[17,18]$. However, these latter findings may be an

253 artefact, because DC sub-populations were analysed as a proportion of total leukocytes, and thus

254 DCs may artificially appear to be reduced because of a larger relative influx of NK cells, T cells

255 and other highly exercise-responsive leukocyte subsets. In a separate study, plasmacytoid and

256 myeloid DCs were examined before and after a combined protocol of moderate aerobic and

257 intermittent resistance exercise undertaken by patients with multiple sclerosis, and healthy 
258 participants [15]. In the aforementioned work, it was shown that myeloid cells increased by $75 \%$

259 and plasmacytoid cells increased by $50 \%$ and there were no differences between patients and

260 healthy controls [15]. Thus, sustained vigorous steady state exercise appears to at least mobilise

261 both myeloid and plasmacytoid DCs, yet we and others [16] have found higher mobilisation

262 responses among plasmacytoid DCs.

263 A preferential mobilisation of plasmacytoid DCs likely represents an adaptive process, in

264 which cells capable of mounting effector responses against infections or cancerous cells are

265 redistributed. Indeed, plasmacytoid DCs are major effector cells in the context of viral infection

266 due to their robust production of type 1 interferons [41, 42]. In addition, these cells express high

267 levels of the toll like receptors TLR7 and TLR9, which transduce signals from virus or self-

268 nucleic acids leading to rapid identification and robust eradication of pathogens [43, 44]. In

269 comparison, myeloid cells are specialised in producing IL-12 that is critical for T cell activation

270 and differentiation $[42,45,46]$. Thus, in an evolutionary context, given that plasmacytoid DCs

271 have a greater inflammatory and migratory potential compared to myeloid DCs [42, 47], it is

272 perhaps unsurprising that these effector cells are preferentially mobilised by exercise.

273 Mechanistically, the magnitude of this mobilisation response is - akin to other effector immune

274 cells preferentially mobilised by acute exercise - likely to be intensity-dependent and driven, in a

275 dose-dependent fashion, by the density of adrenergic receptors on the surface of DCs [48, 49].

276 Indeed, it has been demonstrated that the degree of DC mobilisation during strenuous exercise

277 appears to correlate positively with the concentration of catecholamines released into the

278 peripheral blood [16].

279 Extending previous investigations, we show for the first time that among myeloid DCs,

280 there is a stepwise mobilisation pattern, with the largest responses exhibited by CD1c-CD141- 
281 cells, followed by a decreasing magnitude of response from CD1c+CD141- cells, then

$282 \mathrm{CD} 1 \mathrm{c}+\mathrm{CD} 141+$ cells, with the smallest exercise-induced change exhibited by CD1c-CD141+

283 cells. The least exercise responsive $\mathrm{CD} 1 \mathrm{c}-\mathrm{CD} 141+$ cells identified in the present study,

284 represent a small sub-population of myeloid DCs that have a strong capacity to phagocytose

285 apoptotic and necrotic cells or their debris, cross-presenting antigen to CD8+ cytotoxic T cells

$286[24,50,51]$. In the present study, the two DC sub-populations that mobilised moderately with

287 exercise (CD1c+CD141+ and CD1c+CD141-) both expressed CD1c, and DCs with this

288 characteristic, are potent stimulators of CD4+ T cells [24, 51]. Recently, two additional sub-

289 populations of CD1c+ DCs have been established, referred to as CD1c+_A and CD1c+_B, which

290 exhibit non-inflammatory and inflammatory characteristics respectively [23]. However, as these

291 new sub-populations must be identified by uniquely expressed cell-surface proteins (CD32B for

292 CD1c+_A, and CD163 and CD36 for CD1c+_B), in the present study, we are unable to infer

293 whether the CD1c-expressing cells mobilised by exercise exhibit inflammatory potential [23].

294 Importantly, the present study provides novel information about the least well-characterised

295 myeloid DC sub-population [23]: we show that DCs with a CD1c-CD141- phenotype, are the

296 most exercise-responsive myeloid subset. In addition, future studies may seek to investigate the

297 functional characteristics and homing properties of these cells to better infer the clinical

298 implications of CD1c-CD141- mobilisation during exercise.

299 We also show for the first time, that among myeloid DCs, the most exercise responsive

300 cells are CD205-. For example, CD1c-CD141+ and CD1c+CD141+ cells which did not express

$301 \mathrm{CD} 205$, exhibited an exercise-induced mobilisation that was $80 \%$ and $70 \%$ greater than their

302 CD205+ counterparts, respectively. The cell surface protein CD205 (also known as DEC-205) is

303 upregulated upon DC maturation [25] and facilitates recognition of apoptotic or necrotic cells 
304 [26] by having a critical role in receptor-mediated antigen uptake [52]. Thus, these CD205-

305 cells, which are not specialised in recognising apoptotic or necrotic cells, may have other

306 functions, such as targeting viral infection [53]. In the present study, we also examined DC

307 expression of CD209 (also known as DC-SIGN), a multifunctional receptor which recognises

308 glycans from viruses and bacteria, and is involved in adhesion, migration, signalling and antigen

309 presentation [27]. In agreement with prior research, we showed that DCs in peripheral blood do

310 not express CD209 [13, 54] but we extend these findings by showing that exercise does not

311 stimulate an upregulation of CD209, or at least, does not preferentially mobilise a sub-population

312 of DCs that already express CD209. Circulating DCs become primed to acquire antigens when

313 'activating stimuli' such as cytokines interact with a variety of cell surface receptors [11]. Upon

314 activation, DCs upregulate chemokine receptors, adhesion molecules and co-stimulatory

315 molecules, including CD209 [11]. Indeed, it is well established that developing DCs in vitro for

316 several days with granulocyte-macrophage colony stimulating factor (GM-CSF) and IL-4 leads

317 to an increase in CD209 expression [32, 55]. In addition, inflammatory stimuli such as TNF-

318 alpha, IFN-gamma, and lipopolysaccharide, which also increase with vigorous exercise,

319 upregulate cell surface expression of CD209 [56]. In the present study, it seems that the

320 inflammatory stimulus of exercise was too short, or of insufficient magnitude, to elicit an

321 upregulation of CD209, and thus, these signals might most likely be encountered post-exercise in

322 peripheral tissues, where DCs have been shown to strongly express CD209 [55].

323 The findings presented in this study improve our understanding of how exercise could be

324 used to bolster cell yields for DC immunotherapy [32, 33]. Our results are timely, given the

325 recent focus of harvesting DC sub-populations directly from blood [34, 36, 51, 57]. For example,

326 a feasibility study isolated peripheral blood plasmacytoid DCs from 15 patients with stage IV 
327 melanoma for expansion ex vivo with cytokines and antigen, before re-infusing the cell

328 preparations [58]. The results showed that 7 out of 15 patients were alive two years after

329 plasmacytoid DC administration, compared with 6 of 72 patients treated with standard

330 chemotherapy [58]. Using similar methodology in another feasibility study of 14 patients with

331 stage IV melanoma, immunotherapy using CD1c+ myeloid DCs resulted in long-term

332 progression free survival for 12-35 months [59]. Furthermore, CD1c+ DC immunotherapies are

333 being trialled with other cancers, including prostate cancer [60]. The results of the present study

334 show that if peripheral blood DCs could be harvested during exercise, the total DC yield might

335 increase by $150 \%$, with a potential increased cell yield among plasmacytoid and myeloid

336 dendritic cells by $195 \%$ and $131 \%$ respectively. Depending on the subset of myeloid cells being

337 targeted, exercise could improve cell yields by $23-167 \%$. Future studies are needed to establish

338 whether exercise can increase the yield of peripheral blood DCs in patients with different forms

339 of cancer, and in addition, whether these changes, which might improve the preparation of DC

340 immunotherapy products, leads to better clinical outcomes.

341 The findings of the present study also provide some support for mechanisms underlying

342 vaccine-enhancing effects of acute psychological stress [61] and acute bouts of exercise [29].

343 Indeed, both stressors cause a substantial leukocytosis, and it has been suggested that as part of

344 this response, DCs are mobilised into peripheral blood, later homing to the site of vaccine

345 administration in skeletal muscle, facilitating antigen processing and presentation [62]. Further,

346 the most robust and consistent interventions that enhance vaccine responses induce damage and

347 local inflammation in the muscle selected for vaccine administration [28]. Our study confirms

348 that as part of exercise-induced leukocytosis, DCs are mobilised into peripheral blood, with a

349 preferential response from plasmacytoid cells. Other human studies have shown that leukocytes 
350 appear in muscles damaged by exercise within four to six hours [63, 64]. In support, animal

351 studies have shown that DCs accumulate in damaged muscle within 24 hours [65] perhaps in

352 response to myoblast-derived cytokines and chemokines [66] or heat shock proteins, uric acid

353 and cell debris from necrotic cells [67]. Thus, given the results of the present study and those

354 discussed herein, it is likely that Matzinger's 'danger model', which proposes that antigen

355 presenting cells are attracted to distressed and injured cells, and subsequently activated by

356 endogenous cellular alarm signals, could be a mechanism for improved vaccine responses

357 following muscle-damaging exercise [68]. Further support for this idea is provided by the

358 observation that DCs, and in particular plasmacytoid DCs, are a common feature of lesions in

359 inflammatory myopathies $[69,70]$.

360 When interpreting the results from the present study it should be considered that DCs did

361 not fall below resting levels 30 minutes after exercise. It is likely that the intensity and/or

362 duration of exercise was insufficient to stimulate a post-exercise extravasation of DCs to

363 peripheral tissues [5]. It is well established that a dose-response relationship between exercise

364 duration and the magnitude of lymphocyte trafficking exists, but relationships have not been

365 investigated among DCs [71]. The extravasation of cells out of the bloodstream is likely to be

366 driven by catecholamines and cortisol, and the magnitude of this neuroendocrine response is

367 positively correlated with exercise intensity and duration [72]. In support, a strong positive

368 correlation between adrenergic activity and the exercise-induced increase of plasmacytoid DCs

369 has been reported [16], suggesting an adrenergic dependent mechanism of DC mobilisation, as

370 with other cell populations [73]. However, if in the present study, exercise did indeed invoke a

371 neuroendocrine response of sufficient magnitude, extended blood sampling may have enabled

372 assessment of DC extravasation, given that the post-exercise nadir among lymphocytes is 
373 typically 1-2 hours after the stimulus [3]. To better determine the DC kinetics in response to

374 exercise, future studies should investigate different durations and intensities of exercise with

375 extended post-exercise blood sampling.

376 In summary, acute exercise increased the number of DCs in peripheral blood by $150 \%$

377 with a preferential mobilisation of plasmacytoid DCs (195\%) compared to myeloid DCs (131\%).

378 Among myeloid DCs, there was a stepwise mobilisation pattern: $167 \%$ increase with

379 CD1c-CD141- cells, a 79\% increase with CD1c+CD141-, a 44\% increase with CD1c+CD141+

380 cells and a $23 \%$ increase with $\mathrm{CD} 1 \mathrm{c}-\mathrm{CD} 141+$ cells. The most exercise responsive myeloid DCs

381 did not express CD205, suggesting that immature cells, unspecialised in recognising apoptotic or

382 necrotic cells, are preferentially mobilised.

383

$384 \quad$ Figure legends

385 Figure 1

386 Flow cytometry gating strategy. Doublets were removed by gating forward versus forward-

387 scatter (a). Mononuclear cells were gated on the forward versus side-scatter (b) dead cells were

388 excluded gating 7AAD versus side-scatter (c) followed by subsequent gating of

389 Lineage-HLADR + dendritic cells (d), which were analysed for expression of CD303 (e).

390 Plasmacytoid dendritic cells were identified as Lineage-HLA-DR+CD303+ and myeloid

391 dendritic cells identified as Lineage-HLA-DR + CD303- (e). Myeloid dendritic cells

392 Lineage-HLADR + CD303- were analysed for expression of CD141 and CD1c (f) to yield four

393 sub-populations CD1c-CD141+; CD1c+CD141+; CD1c+CD141-; CD1c-CD141- (g). CD205

394 gating was determined using fluorescence-minus-one (FMO) tubes and applied to all cell

395 populations (h). 
Figure 2

398 Mobilisation of total dendritic cells and subpopulations during exercise. (a) Exercise-induced

399 kinetics of dendritic cells. Main effect of time: $F_{(1,10)}=60.0 ; p<0.05 \eta^{2}=0.9 .{ }^{*}$ Indicates a 400 significant difference from baseline $\mathrm{p}<0.05$ ( $t$-test between baseline and exercise; $\mathrm{t}_{(8)}=-6.9$, $401 p<0.05)$ and a significant difference from $+30 \min \left(t\right.$-test between exercise and $+30 \mathrm{~min} ; \mathrm{t}_{(8)}=$ 402 14.2, $\mathrm{p}<0.05)$. Data are expressed as cell $/ \mu \mathrm{L}$ (mean \pm SEM). (b) Percentage change from baseline 403 for major dendritic cell subsets in response to exercise. * Indicates a significant difference 404 between subsets $\mathrm{p}<0.05$ ( $t$-test; Plasmacytoid $v s$. Myeloid, $\left.\mathrm{t}_{(8)}=-2.9, p<0.05\right)$. Data are 405 expressed as percentage change from baseline (mean \pm SEM). (c) Percentage change for myeloid 406 dendritic cell sub-populations in response to exercise. * Indicates a significant difference

407 between subsets $\mathrm{p}<0.05$ ( $t$-test; $\mathrm{CD} 1 \mathrm{c}-\mathrm{CD} 141+v_{s} \mathrm{CD} 1 \mathrm{c}-\mathrm{CD} 141-, \mathrm{t}_{(8)}=-3.0, p<0.05$;

$408 \mathrm{CD} 1 \mathrm{c}+\mathrm{CD} 141+v_{s} \mathrm{CD} 1 \mathrm{c}-\mathrm{CD} 141-\mathrm{t}_{(8)}=-3.1, p<0.05 ; \mathrm{CD} 1 \mathrm{c}+\mathrm{CD} 141-v s \mathrm{CD} 1 \mathrm{c}-\mathrm{CD} 141-, \mathrm{t}_{(8)}=$ $409-3.9, p<0.05)$. No other significant differences were observed between cell types. Data are 410 expressed as percentage change from baseline (mean \pm SEM).

411

\section{Figure 3}

413 Differential magnitude of dendritic cell and subpopulation mobilisation on the basis of CD205 414 expression. Percentage change from baseline for major dendritic cell subsets and the myeloid 415 dendritic cell sub-populations in response to exercise. * Indicates a significant difference 416 between $\mathrm{CD} 205+$ and $\mathrm{CD} 205-\mathrm{p}<0.05 t$-test. $\mathrm{CD} 1 \mathrm{c}-\mathrm{CD} 141+, \mathrm{t}_{(8)}=-2.5, p<0.05$;

$417 \mathrm{CD} 1 \mathrm{c}+\mathrm{CD} 141+, \mathrm{t}_{(8)}=-3.1, p<0.05$. Data are expressed as percentage change from baseline 418 (mean \pm SEM). 


\section{References}

420

421

422

423

424

425

426

427

428

429

430

431

432

433

434

435

436

437

438

439

440

441

442

443

444

445

446

447

448

449

450

451

452

453

454

455

456

457

458

459

460

461

462

463

464

465

466

467

468

469

470

471

472

473

1. Walsh, N.P., et al., Position statement. Part one: Immune function and exercise. Exerc Immunol Rev, 2011. 17: p. 6-63.

2. Campbell, J.P., et al., Acute exercise mobilises CD8+ T lymphocytes exhibiting an effector-memory phenotype. Brain, Behavior, and Immunity, 2009. 23(6): p. 767-75.

3. Simpson, R.J., et al., Senescent T-lymphocytes are mobilised into the peripheral blood compartment in young and older humans after exhaustive exercise. Brain, Behavior, and Immunity, 2008. 22(4): p. 544-51.

4. Kruger, K., et al., Exercise-induced redistribution of T lymphocytes is regulated by adrenergic mechanisms. Brain, Behavior, and Immunity, 2008. 22(3): p. 324-38.

5. Dhabhar, F.S., et al., Stress-induced redistribution of immune cells--from barracks to boulevards to battlefields: a tale of three hormones--Curt Richter Award winner. Psychoneuroendocrinology, 2012. 37(9): p. 1345-68.

6. $\quad$ Brown, F.F., et al., Training status and sex influence on senescent T-lymphocyte redistribution in response to acute maximal exercise. Brain Behav Immun, 2014. 39: p. 152-9.

7. Bigley, A.B., et al., Acute exercise preferentially redeploys NK-cells with a highly-differentiated phenotype and augments cytotoxicity against lymphoma and multiple myeloma target cells. Brain Behav Immun, 2014. 39: p. 160-71.

8. Booth, S., et al., The impact of acute strenuous exercise on TLR2, TLR4 and HLA.DR expression on human blood monocytes induced by autologous serum. Eur J Appl Physiol, 2010. 110(6): p. 1259-68.

9. Simpson, R.J., et al., Toll-like receptor expression on classic and pro-inflammatory blood monocytes after acute exercise in humans. Brain Behav Immun, 2009. 23(2): p. 232-9.

10. Hong, S. and P.J. Mills, Effects of an Exercise Challenge on Mobilization and Surface Marker Expression of Monocyte Subsets in Individuals with Normal vs. Elevated Blood Pressure. Brain, behavior, and immunity, 2008. 22(4): p. 590-599.

11. Merad, M., et al., The dendritic cell lineage: ontogeny and function of dendritic cells and their subsets in the steady state and the inflamed setting. Annu Rev Immunol, 2013. 31: p. 563-604.

12. Haniffa, M., V. Bigley, and M. Collin, Human mononuclear phagocyte system reunited. Semin Cell Dev Biol, 2015. 41: p. 59-69.

13. MacDonald, K.P., et al., Characterization of human blood dendritic cell subsets. Blood, 2002. 100(13): p. 4512-20.

14. Ho, C.S.K., et al., Surgical and physical stress increases circulating blood dendritic cell counts independently of monocyte counts. Blood, 2001. 98(1): p. 140-145.

15. Deckx, N., et al., Rapid Exercise-Induced Mobilization of Dendritic Cells Is Potentially Mediated by a Flt3L- and MMP-9-Dependent Process in Multiple Sclerosis. Mediators Inflamm, 2015. 2015: $\mathrm{p}$. 158956.

16. Suchánek, O., et al., Intensive physical activity increases peripheral blood dendritic cells. Cell Immunol, 2010. 266(1): p. 40-5.

17. Nickel, T., et al., Modulation of dendritic cells and toll-like receptors by marathon running. Eur $\mathrm{J}$ Appl Physiol, 2012. 112(5): p. 1699-708.

18. Lackermair, K., et al., Influence of polyphenol-rich diet on exercise-induced Immunomodulation in male endurance athletes. Appl Physiol Nutr Metab, 2017.

19. Ziegler-Heitbrock, L., et al., Nomenclature of monocytes and dendritic cells in blood. Blood, 2010. 116(16): p. e74-80.

20. Dzionek, A., et al., BDCA-2, BDCA-3, and BDCA-4: three markers for distinct subsets of dendritic cells in human peripheral blood. J Immunol, 2000. 165(11): p. 6037-46.

21. Penna, G., et al., Cutting Edge: Differential Chemokine Production by Myeloid and Plasmacytoid Dendritic Cells. The Journal of Immunology, 2002. 169(12): p. 6673-6676.

22. Thomas, R. and P.E. Lipsky, Human peripheral blood dendritic cell subsets. Isolation and characterization of precursor and mature antigen-presenting cells. J Immunol, 1994. 153(9): p. 4016-28.

23. Villani, A.C., et al., Single-cell RNA-seq reveals new types of human blood dendritic cells, monocytes, and progenitors. Science, 2017. 356(6335). 
24. Ding, Y., et al., FLT3-ligand treatment of humanized mice results in the generation of large numbers of $C D 141+$ and $C D 1 c+$ dendritic cells in vivo. J Immunol, 2014. 192(4): p. 1982-9.

25. Butler, M., et al., Altered expression and endocytic function of CD205 in human dendritic cells, and detection of a CD205-DCL-1 fusion protein upon dendritic cell maturation. Immunology, 2007. 120(3): p. 362-71.

26. Cao, L., et al., $\mathrm{pH}$-Dependent recognition of apoptotic and necrotic cells by the human dendritic cell receptor DEC205. Proc Natl Acad Sci U S A, 2015. 112(23): p. 7237-42.

27. Garcia-Vallejo, J.J. and Y. van Kooyk, The physiological role of DC-SIGN: a tale of mice and men. Trends Immunol, 2013. 34(10): p. 482-6.

28. Pascoe, A.R., M.A. Fiatarone Singh, and K.M. Edwards, The effects of exercise on vaccination responses: a review of chronic and acute exercise interventions in humans. Brain Behav Immun, 2014. 39: p. 33-41.

29. Edwards, K.M., et al., Eccentric exercise as an adjuvant to influenza vaccination in humans. Brain Behav Immun, 2007. 21(2): p. 209-17.

30. Edwards, K.M., et al., Exercise intensity does not influence the efficacy of eccentric exercise as a behavioural adjuvant to vaccination. Brain Behav Immun, 2010. 24(4): p. 623-30.

31. Ranadive, S.M., et al., Effect of acute aerobic exercise on vaccine efficacy in older adults. Med Sci Sports Exerc, 2014. 46(3): p. 455-61.

32. LaVoy, E.C., et al., $A$ single bout of dynamic exercise by healthy adults enhances the generation of monocyte-derived-dendritic cells. Cell Immunol, 2015. 295(1): p. 52-9.

33. Simpson, R.J., et al., Mobilizing Immune Cells with Exercise for Cancer Immunotherapy. Exerc Sport Sci Rev, 2017.

34. Garg, A.D., et al., Integrating Next-Generation Dendritic Cell Vaccines into the Current Cancer Immunotherapy Landscape. Trends Immunol, 2017. 38(8): p. 577-593.

35. Garg, A.D., et al., Trial watch: Dendritic cell-based anticancer immunotherapy. Oncoimmunology, 2017. 6(7): p. e1328341.

36. Sabado, R.L., S. Balan, and N. Bhardwaj, Dendritic cell-based immunotherapy. Cell Res, 2017. 27(1): p. 74-95.

37. Wadley, A.J., et al., Low volume-high intensity interval exercise elicits antioxidant and antiinflammatory effects in humans. J Sports Sci, 2016. 34(1): p. 1-9.

38. Wadley, A.J., et al., Monitoring changes in thioredoxin and over-oxidised peroxiredoxin in response to exercise in humans. Free Radic Res, 2015. 49(3): p. 290-8.

39. Turner, J.E., et al., Intensive Exercise Does Not Preferentially Mobilize Skin-Homing T Cells and NK Cells. Med Sci Sports Exerc, 2016. 48(7): p. 1285-93.

40. Turner, J.E., et al., Exercise-induced B cell mobilisation: Preliminary evidence for an influx of immature cells into the bloodstream. Physiol Behav, 2016. 164(Pt A): p. 376-82.

41. McKenna, K., A.S. Beignon, and N. Bhardwaj, Plasmacytoid dendritic cells: linking innate and adaptive immunity. J Virol, 2005. 79(1): p. 17-27.

42. Liu, Y.J., IPC: professional type 1 interferon-producing cells and plasmacytoid dendritic cell precursors. Annu Rev Immunol, 2005. 23: p. 275-306.

43. Lande, R., et al., Plasmacytoid dendritic cells sense self-DNA coupled with antimicrobial peptide. Nature, 2007. 449(7162): p. 564-9.

44. Gilliet, M., W. Cao, and Y.J. Liu, Plasmacytoid dendritic cells: sensing nucleic acids in viral infection and autoimmune diseases. Nat Rev Immunol, 2008. 8(8): p. 594-606.

45. Heufler, C., et al., Interleukin-12 is produced by dendritic cells and mediates $T$ helper 1 development as well as interferon-gamma production by Thelper 1 cells. Eur J Immunol, 1996. 26(3): p. 659-68.

46. Zundler, S. and M.F. Neurath, Interleukin-12: Functional activities and implications for disease. Cytokine Growth Factor Rev, 2015. 26(5): p. 559-68.

47. Cox, K., et al., Plasmacytoid dendritic cells (PDC) are the major DC subset innately producing cytokines in human lymph nodes. J Leukoc Biol, 2005. 78(5): p. 1142-52.

48. Maestroni, G.J., Dendritic cell migration controlled by alpha $1 \mathrm{~b}$-adrenergic receptors. J Immunol, 2000. 165(12): p. 6743-7.

49. Nijhuis, L.E., et al., Adrenergic $\beta 2$ receptor activation stimulates anti-inflammatory properties of dendritic cells in vitro. PLoS One, 2014. 9(1): p. e85086. 
50. Haniffa, M., et al., Human tissues contain CD141hi cross-presenting dendritic cells with functional homology to mouse CD103+ nonlymphoid dendritic cells. Immunity, 2012. 37(1): p. 60-73.

51. Jongbloed, S.L., et al., Human CD141+ (BDCA-3)+ dendritic cells (DCs) represent a unique myeloid DC subset that cross-presents necrotic cell antigens. J Exp Med, 2010. 207(6): p. 1247-60.

52. Mahnke, K., et al., The dendritic cell receptor for endocytosis, DEC-205, can recycle and enhance antigen presentation via major histocompatibility complex class II-positive lysosomal compartments. J Cell Biol, 2000. 151(3): p. 673-84.

53. Kato, M., et al., Expression of human DEC-205 (CD205) multilectin receptor on leukocytes. Int Immunol, 2006. 18(6): p. 857-69.

54. Piccioli, D., et al., Functional specialization of human circulating CD16 and CD1c myeloid dendriticcell subsets. Blood, 2007. 109(12): p. 5371-9.

55. Geijtenbeek, T.B., et al., Identification of DC-SIGN, a novel dendritic cell-specific ICAM-3 receptor that supports primary immune responses. Cell, 2000. 100(5): p. 575-85.

56. Fung, E., et al., Multiplexed immunophenotyping of human antigen-presenting cells in whole blood by polychromatic flow cytometry. Nat Protoc, 2010. 5(2): p. 357-70.

57. Sittig, S.P., et al., A Comparative Study of the T Cell Stimulatory and Polarizing Capacity of Human Primary Blood Dendritic Cell Subsets. 2016. 2016: p. 3605643.

58. Tel, J., et al., Natural human plasmacytoid dendritic cells induce antigen-specific T-cell responses in melanoma patients. Cancer Res, 2013. 73(3): p. 1063-75.

59. Schreibelt, G., et al., Effective Clinical Responses in Metastatic Melanoma Patients after Vaccination with Primary Myeloid Dendritic Cells. Clin Cancer Res, 2016. 22(9): p. 2155-66.

60. Prue, R.L., et al., A phase I clinical trial of CD1C (BDCA-1)+ dendritic cells pulsed with HLA-A*0201 peptides for immunotherapy of metastatic hormone refractory prostate cancer. J Immunother, 2015. 38(2): p. 71-6.

61. Edwards, K.M., et al., Acute stress exposure prior to influenza vaccination enhances antibody response in women. Brain Behav Immun, 2006. 20(2): p. 159-68.

62. Edwards, K.M., et al., The acute stress-induced immunoenhancement hypothesis. Exerc Sport Sci Rev, 2007. 35(3): p. 150-5.

63. Malm, C., et al., Immunological changes in human skeletal muscle and blood after eccentric exercise and multiple biopsies. J Physiol, 2000. 529 Pt 1: p. 243-62.

64. Paulsen, G., et al., Time course of leukocyte accumulation in human muscle after eccentric exercise. Med Sci Sports Exerc, 2010. 42(1): p. 75-85.

65. Wirsdörfer, F., et al., Dendritic Cell-Like Cells Accumulate in Regenerating Murine Skeletal Muscle after Injury and Boost Adaptive Immune Responses Only upon a Microbial Challenge. PLoS One, 2016. 11(5): p. e0155870.

66. De Rossi, M., et al., Cytokines and chemokines are both expressed by human myoblasts: possible relevance for the immune pathogenesis of muscle inflammation. Int Immunol, 2000. 12(9): p. 132935.

67. Shi, Y. and K.L. Rock, Cell death releases endogenous adjuvants that selectively enhance immune surveillance of particulate antigens. Eur J Immunol, 2002. 32(1): p. 155-62.

68. Matzinger, P., The danger model: a renewed sense of self. Science, 2002. 296(5566): p. 301-5.

69. Tateyama, M., K. Fujihara, and Y. Itoyama, Dendritic cells in muscle lesions of sarcoidosis. Hum Pathol, 2011. 42(3): p. 340-6.

70. López de Padilla, C.M., et al., Plasmacytoid dendritic cells in inflamed muscle of patients with juvenile dermatomyositis. Arthritis Rheum, 2007. 56(5): p. 1658-68.

71. Hansen, J.-B., L. Wilsgård, and B. Østerud, Biphasic changes in leukocytes induced by strenuous exercise. European Journal of Applied Physiology and Occupational Physiology, 1991. 62(3): p. 157-161.

72. Hill, E.E., et al., Exercise and circulating Cortisol levels: The intensity threshold effect. Journal of Endocrinological Investigation, 2008. 31(7): p. 587-591.

73. Elenkov, I.J., et al., The sympathetic nerve--an integrative interface between two supersystems: the brain and the immune system. Pharmacol Rev, 2000. 52(4): p. 595-638. 
Table 1. Dendritic cell sub-population identification

\begin{tabular}{|c|c|c|c|}
\hline Sub-population name & Cell surface markers & Functional properties & Reference \\
\hline DCs & Lineage- HLADR+ & $\begin{array}{l}\text { Presentation of ingested pathogens or cell debris to } \\
\text { T-cells. }\end{array}$ & $\begin{array}{l}\text { Ziegler Heitbrock (2010) } \\
\text { Merad (2013) }\end{array}$ \\
\hline Plasmacytoid DCs & $\begin{array}{l}\text { Lineage }- \text { HLADR }+ \\
\text { CD303+ }\end{array}$ & $\begin{array}{l}\text { Major effector sub-population of DCs. Produce type } \\
1 \text { interferons in response to viral infection. }\end{array}$ & $\begin{array}{l}\text { Dzionek (2000) } \\
\text { Liu (2005) }\end{array}$ \\
\hline Myeloid DCs & $\begin{array}{l}\text { Lineage- HLADR }+ \\
\text { CD303- }\end{array}$ & $\begin{array}{l}\text { Regulatory DC sub-populations. Produce IL-12 for } \\
\text { T-cell activation and differentiation. }\end{array}$ & $\begin{array}{l}\text { Dzionek (2000) } \\
\text { Heufler (1996) }\end{array}$ \\
\hline CD1c-CD141+ & $\begin{array}{l}\text { Lineage- HLADR }+ \\
\text { CD303- CD1c-CD141+ }\end{array}$ & $\begin{array}{l}\text { Cross presentation of antigen to CD } 8+\mathrm{T} \text {-cells for } \\
\text { anti-tumour immunity. }\end{array}$ & $\begin{array}{l}\text { Penna (2002) } \\
\text { Ding (2014) }\end{array}$ \\
\hline CD1c+CD141+ & $\begin{array}{l}\text { Lineage- HLADR }+ \\
\text { CD303-CD1c+CD141+ }\end{array}$ & $\begin{array}{l}\text { Cross presentation of antigen to CD8+ T cells for } \\
\text { anti-tumour immunity and stimulate CD4+ T-cells. }\end{array}$ & $\begin{array}{l}\text { Villani (2017) } \\
\text { Ding (2014) }\end{array}$ \\
\hline CD1c+CD141- & $\begin{array}{l}\text { Lineage- HLADR+ } \\
\text { CD303- CD1c+ CD141- }\end{array}$ & Stimulate CD4+ T-cells. & $\begin{array}{l}\text { Villani (2017) } \\
\text { Ding (2014) }\end{array}$ \\
\hline CD1c-CD141- & $\begin{array}{l}\text { Lineage- HLADR+ } \\
\text { CD303-CD1c-CD141- }\end{array}$ & Unknown & Villani (2017) \\
\hline
\end{tabular}

Legend: Indentation indicates a sub-population of parent cells (i.e., Myeloid Dendritic Cells are a sub-population of Dendritic Cells, and CD1c $+C D 141-$ Dendritic Cells are a sub-population of Myeloid Dendritic Cells). Lineage cocktail $=$ CD3, CD19, CD20, CD14, CD56. HLADR = marker for major histocompatibility complex MHC class II. CD = cluster of differentiation. In addition CD205 (DEC-205) a cell surface marker that enables recognition of apoptotic or necrotic cells (Cao et al 2015) and CD209 (DC-SIGN) a cell surface marker that recognises a wide variety of ligands, is involved in adhesion, migration and antigen presentation (Garcia-Vallejo and van Kooyk 2013) were examined on all dendritic cells and sub-populations. 
Table 2. Total DCs, and DC sub-populations differentiated on CD205 expression (mean \pm SD)

\begin{tabular}{|c|c|c|c|c|c|}
\hline Cells $/ \mu \mathrm{L}$ & & Baseline & Exercise & $+30 \mathrm{~min}$ & Main effect of time \\
\hline \multirow[t]{3}{*}{ DCs } & & $79 \pm 38$ & $196 \pm 126^{* * *}$ & $76 \pm 34 \dagger$ & $F_{(1,10)}=59.9 ; p<0.05 \eta^{2}=0.9$ \\
\hline & CD205+ & $78 \pm 37$ & $192 \pm 123^{* * *}$ & $74 \pm 33 \dagger$ & $F_{(1,10)}=59.9 ; p<0.05 \eta^{2}=0.9$ \\
\hline & CD205- & $1.4 \pm 1.4$ & $4.3 \pm 4.8^{* * *}$ & $1.5 \pm 1.5 \dagger$ & $F_{(2,16)}=27.8 ; p<0.05 \eta^{2}=0.8$ \\
\hline \multirow[t]{3}{*}{ Plasmacytoid DCs } & & $19 \pm 11$ & $55 \pm 42^{* * *}$ & $22 \pm 19 \dagger$ & $F_{(2,16)}=45.9 ; p<0.05 \eta^{2}=0.9$ \\
\hline & CD205+ & $19 \pm 11$ & $55 \pm 42^{* * *}$ & $22 \pm 19 \dagger$ & $F_{(2,16)}=45.9 ; p<0.05 \eta^{2}=0.9$ \\
\hline & CD205- & $0.07 \pm 0.07$ & $0.1 \pm 0.09^{* *}$ & $0.06 \pm 0.03$ & $F_{(2,16)}=5.0 ; p<0.05 \eta^{2}=0.4$ \\
\hline \multirow[t]{3}{*}{ Myeloid DCs } & & $60 \pm 30$ & $139 \pm 88^{* * *}$ & $53 \pm 22 \dagger$ & $F_{(2,16)}=43.6 ; p<0.05 \eta^{2}=0.8$ \\
\hline & CD205+ & $58 \pm 30$ & $135 \pm 85^{* * *}$ & $52 \pm 21 \dagger$ & $F_{(2,16)}=43.4 ; p<0.05 \eta^{2}=0.8$ \\
\hline & CD205- & $1.4 \pm 1.3$ & $4.2 \pm 4.7^{* * *}$ & $1.4 \pm 1.5 \dagger$ & $F_{(2,16)}=28.1 ; p<0.05 \eta^{2}=0.8$ \\
\hline \multirow[t]{3}{*}{ CD1c-CD141+ } & & $2.1 \pm 1.3$ & $2.4 \pm 1.5$ & $1.7 \pm 0.9$ & $F_{(2,16)}=1.4 ; p>0.05 \eta^{2}=0.2$ \\
\hline & CD205+ & $2.0 \pm 1.3$ & $2.2 \pm 1.5$ & $1.6 \pm 0.9$ & $F_{(2,16)}=1.5 ; p>0.05 \eta^{2}=0.2$ \\
\hline & CD205- & $0.08 \pm 0.1$ & $0.1 \pm 0.2$ & $0.09 \pm 0.1$ & $F_{(2,16)}=3.6 ; p>0.05 \eta^{2}=0.3$ \\
\hline \multirow[t]{3}{*}{ CD1c+ CD141+ } & & $0.6 \pm 0.2$ & $0.8 \pm 0.4^{* *}$ & $0.6 \pm 0.2$ & $F_{(2,16)}=5.1 ; p<0.05 \eta^{2}=0.4$ \\
\hline & CD205+ & $0.54 \pm 0.2$ & $0.78 \pm 0.38^{*}$ & $0.54 \pm 0.2$ & $F_{(2,16)}=4.6 ; p<0.05 \eta^{2}=0.4$ \\
\hline & CD205- & $0.01 \pm 0.01$ & $0.03 \pm 0.01 * * *$ & $0.01 \pm 0.01 \dagger$ & $F_{(2,16)}=21.3 ; p<0.05 \eta^{2}=0.7$ \\
\hline \multirow[t]{3}{*}{ CD1c+ CD141- } & & $20 \pm 12$ & $34 \pm 18^{* * *}$ & $16 \pm 6 \dagger$ & $F_{(2,16)}=31.2 ; p<0.05 \eta^{2}=0.8$ \\
\hline & CD205+ & $20 \pm 12$ & $33.7 \pm 17.4^{* * *}$ & $16 \pm 6 \dagger$ & $F_{(2,16)}=31.1 ; p<0.05 \eta^{2}=0.8$ \\
\hline & CD205- & $0.07 \pm 0.08$ & $0.14 \pm 0.16$ & $0.04 \pm 6 \dagger$ & $F_{(2,16)}=3.9 ; p<0.05 \eta^{2}=0.3$ \\
\hline \multirow[t]{3}{*}{ CD1c- CD141- } & & $37 \pm 22$ & $102 \pm 78^{* * *}$ & $35 \pm 21 \dagger$ & $F_{(2,16)}=45.2 ; p<0.05 \eta^{2}=0.9$ \\
\hline & CD205+ & $35.7 \pm 21.3$ & $98.1 \pm 74^{* * *}$ & $33.3 \pm 19.6 \dagger$ & $F_{(2,16)}=45.2 ; p<0.05 \eta^{2}=0.9$ \\
\hline & CD205- & $1.3 \pm 1.1$ & $4 \pm 4.4^{* * *}$ & $1.3 \pm 1.3 \dagger$ & $F_{(2,16)}=27.1 ; p<0.05 \eta^{2}=0.8$ \\
\hline
\end{tabular}

Legend: $*<0.05 * *<0.01 * * *<0.001$ indicates a significant difference (paired samples t-test from baseline). $\dagger$ indicates a significant difference $<0.05$ (paired samples t-test from exercise to $+30 \mathrm{~min}$ ). 
Figure 1 . Flow cytometry gating strategy

a
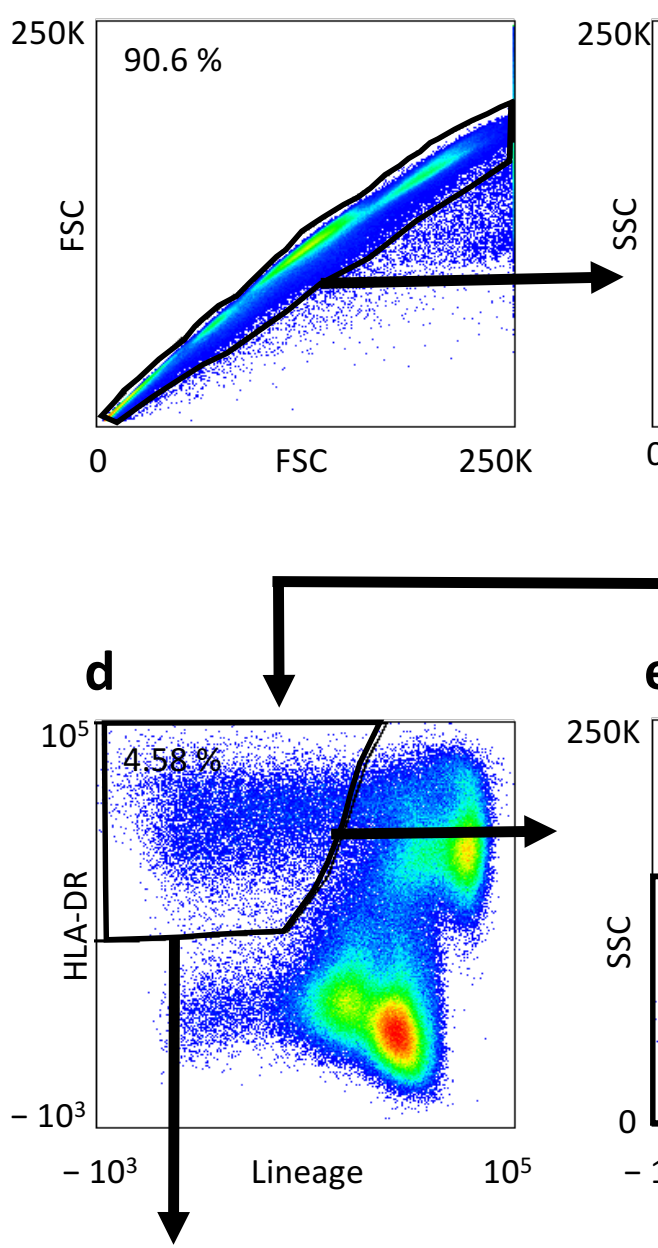

h CD205 gating

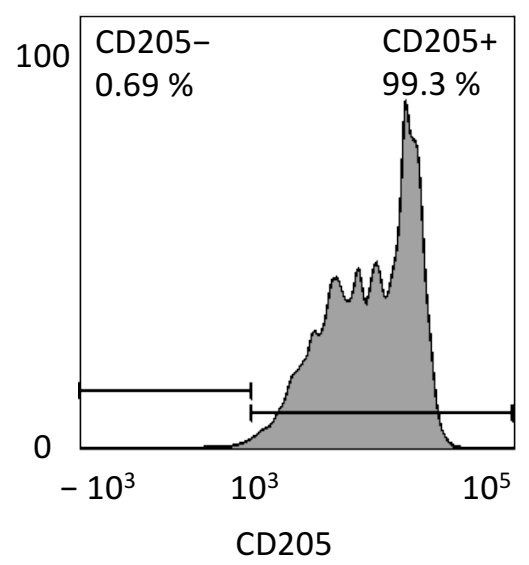

b

e

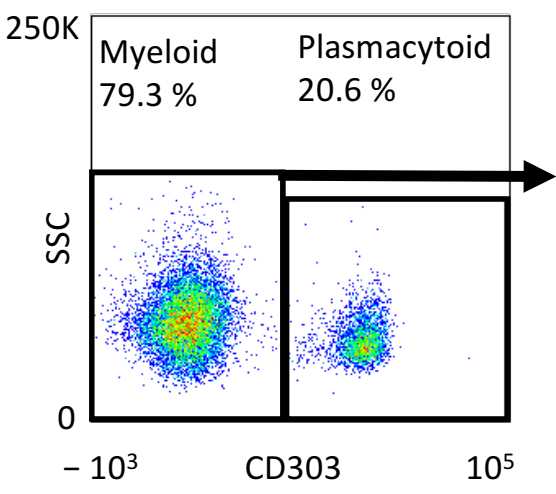

$-10^{3}$

CD303

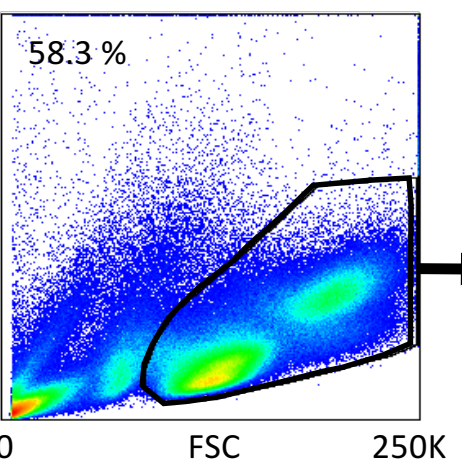

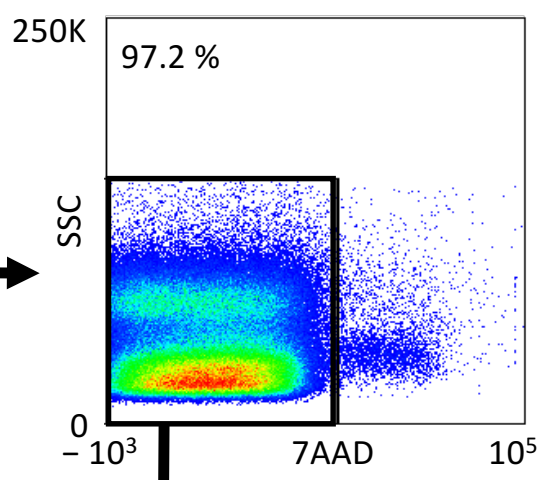

f

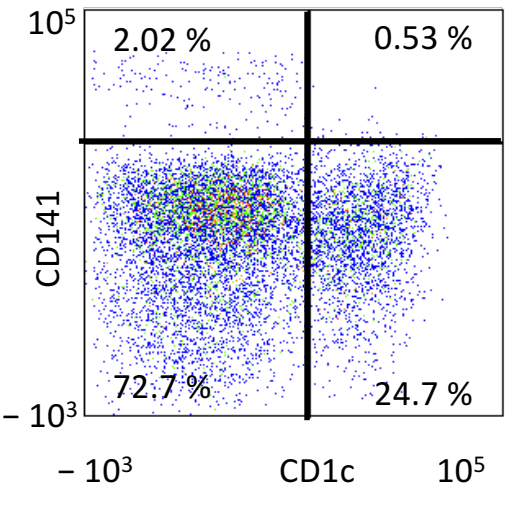

g Subset identification

\begin{tabular}{|l|l|}
\hline $\begin{array}{l}\text { CD1c- } \\
\text { CD141+ }\end{array}$ & $\begin{array}{l}\text { CD1C+ } \\
\mathrm{CD} 141+\end{array}$ \\
\hline & \\
CD1c- & CD1c+ \\
CD141- & CD141- \\
& \\
\hline
\end{tabular}


Figure 2. Mobilisation of total dendritic cells and sub-populations during exercise
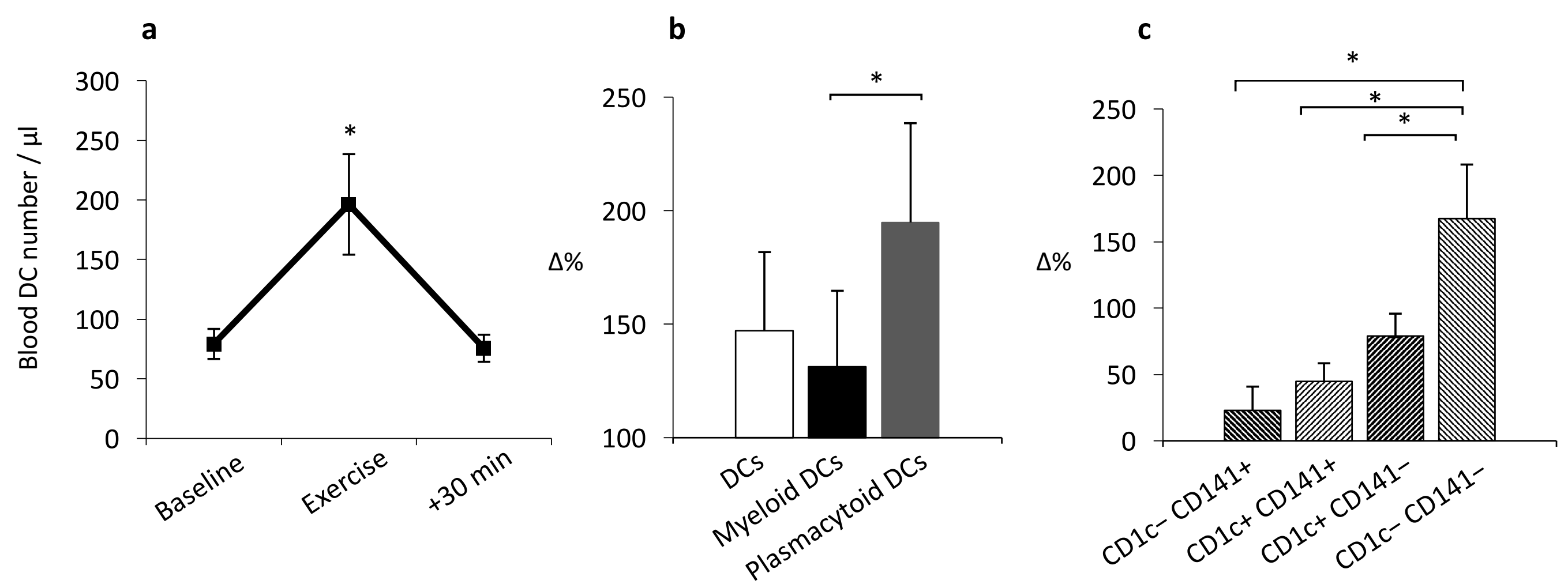
Figure 3. Differential magnitude of dendritic cell and sub-population mobilisation on the basis of CD205 expression
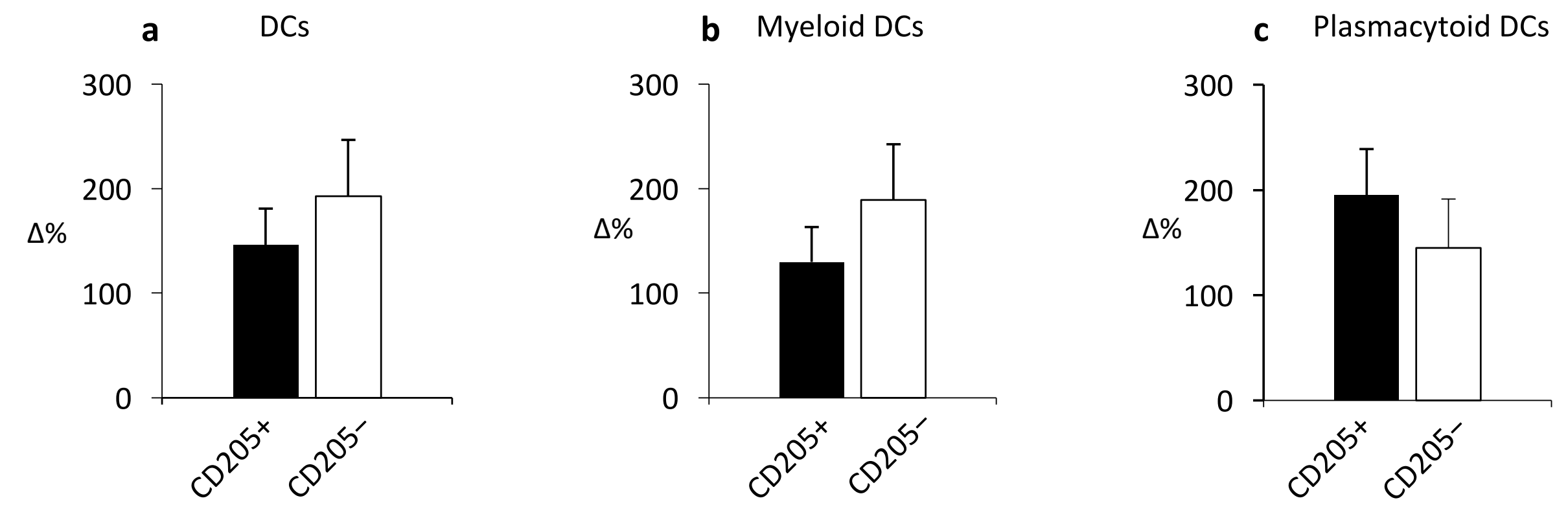

d CD1c-CD141+

e $\operatorname{CD} 1 \mathrm{c}+\mathrm{CD} 141+$

f $C D 1 c+C D 141-$

g CD1C-CD141-
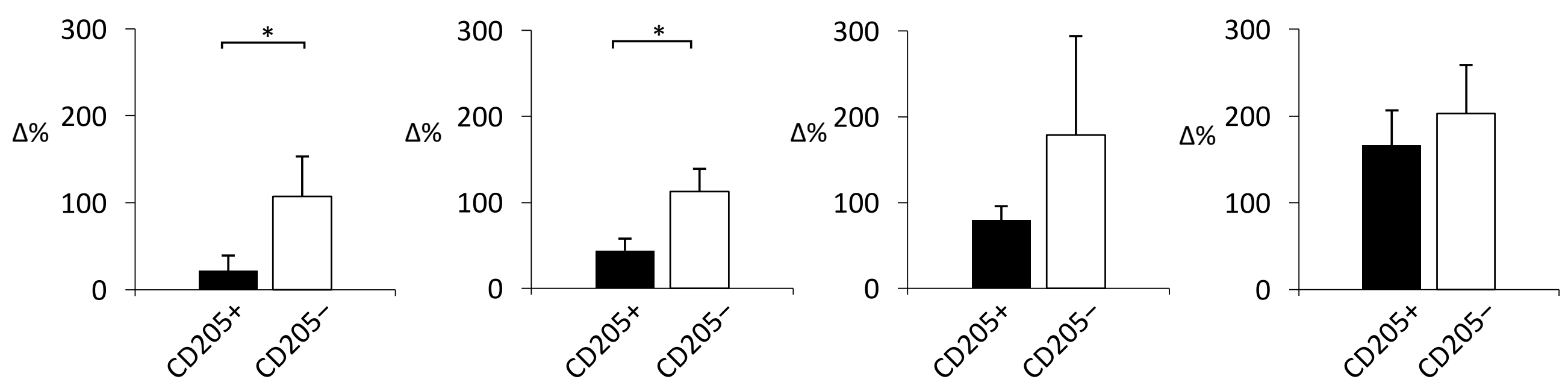